\title{
UN MIEMBRO PROACTIVO DE LA INVESTIGACIÓN EN LA FAMURP: SOCEMURP
}

\author{
A PROACTIVE MEMBER OF THE RESEARCH IN FAMURP : SOCEMURP \\ Cvetkovic-Vega A 1,2,3, a , Mejía CR 2,4, b , Patiño-Calla Karina 2, b , Diana Llacta-Aparicio 2, b, \\ Cesar Espinoza-Chiong 1,2,a, Mariela Vargas 1,2,a, Katherine M. García-Moreno 1,2, a
}

\section{Sr. Editor}

Uno de los fines de la Universidad es la investigación, siendo inclusive considerada por algunos entendidos como la función central de ésta'. En nuestro caso la Universidad Ricardo Palma, fundado el 1 ero de julio de 1969, enuncia en medios oficiales su dedicación entre otros aspectos a la investigación y a través de sus facultades e institutos de investigación desarrolla lineamientos y políticas que fomenten la generación de nuevos conocimientos.

La facultad de Medicina Humana, FAMURP, fue creada el 2 de octubre de 1998, introduciendo cursos de metodología de la investigación en su malla curricular para fomentar la investigación en el pre-grado. Diez años después de su creación, en el 2008 fue creado el Instituto de Investigación en Ciencias Biomédicas, INICIB, para impulsar el desarrollo de líneas de investigación y colaboración entre docentes y los estudiantes interesados en investigar.

Siguiendo diferentes ejemplos de estudiantes de medicina a lo largo del Perú y que desde el 27 de agosto de 1992 se congregan a través la Sociedad Científica Médico Estudiantil Peruana-SOCIMEP, y que a la fecha congrega a 37 Sociedades Científicas en 15 regiones del Perú, los estudiantes de medicina de la Universidad Ricardo Palma reunidos en Asamblea y con aprobación de las autoridades universitarias crearon el 6 de julio del 2005 la Sociedad Científica de Estudiantes de Medicina de la Universidad Ricardo Palma -SOCEMURP- la cual en el año 2009 quedaría adscrita al INICIB para canalizar las iniciativas de investigación estudiantil bajo los lineamientos de investigación de la FAMURP. Actualmente, SOCEMURP tiene representación nacional como miembro titular de SOCIMEP y un alumno de sexto año de Medicina de la FAMURP, el Univ. Aleksandar Cvetkovic, se desempeña como Presidente en la gestión 2015-2016; además, SOCEMURP a través de SOCIMEP participa de la Federación Latinoamericana de Sociedades Científicas, FELSOCEM, y desde el 2013 es miembro asociado de la International Federation of Medical Students Association, IFMSA.

En el 2015, SOCEMURP celebró su décimo aniversario con diversos logros científicos que engrandecen el nombre de nuestra universidad: 10 publicaciones científicas con filiación URP en revistas indizadas lo cual ha contribuye a mejorar el puesto la URP en los rankings universitarios que clasifican a las universidades de acuerdo a su producción científica en SCOPUS²; la organización del Campamento Universitario Multidisciplinario de Investigación y Salud (CUMIS), denominado CUMIS Nacional Sicaya 2015; el primer puesto en trabajos de investigación en el Congreso Científico Nacional de Estudiantes de Medicina (CCN) Iquitos 2015; el primer estudiante de la URP electo para el cargo de Presidente de SOCIMEP, y el nombramiento de SOCEMURP como la mejor SOCEM del 2015 lo cual es una nominación honrosa otorgada por SOCIMEP de entre 37 SOCEMs a nivel del Perú.

Agruparse en SOCEMs permiten la adquisición de diferentes competencias ${ }^{3}$ con componentes tales como la educación médica, salud pública, movilidad internacional, administración y proyección, redes de trabajo y cultura, el fortalecimiento de capacidades científicas, y publicación científica y formación editorial. La investigación se aprende investigando y teniendo modelos a seguir ${ }^{4}$; Arce y

\footnotetext{
Facultad de Medicina Humana, Universidad Ricardo Palma, Lima, Perú.

2. Sociedad Científica de Estudiantes de Medicina de la Universidad Ricardo Palma, SOCEMURP.

3. Sociedad Científica Médico Estudiantil Peruana, SOCIMEP.

4. Asociación Médica de Investigación y Servicios de Salud, Lima, Perú.

astudiante de Medicina

b. Médico Cirujano.
}

\author{
Correspondencia: \\ Aleksandar Cvetkovic V. \\ Dirección: \\ Broncino 307, San Borja, Lima, Perú \\ Teléfono: \\ +511964982676 \\ Correo electrónico: \\ aleksandar.famurp@gmail.com
}


Astuvilca plantearon en el 2007 la selección de estudiantes comprometidos y motivados para capacitarlos en aspectos básicos de metodología de la investigación, pudiendo así efectuar un acercamiento con los institutos de investigación de las facultades y buscar mentores médicos-científicos ${ }^{5}$. La realidad es que en la FAMURP estás 2 estrategias sugeridas ya se han implementado, sin embargo sigue siendo un reto la búsqueda de mentores y el desarrollo de líneas de investigación con inclusión de alumnos capacitados para investigar y cuya producción cien- tífica sea visible en revistas indizadas a SCOPUS ${ }^{6}$.

Fuente de financiamiento: Autofinanciado.

Conflicto de interés: Los autores declaran no tener conflictos de interés en la publicación de este artículo.

Recibido: 28/03/2016

Aprobado: 08/04/2016

\section{REFERENCIAS BIBLIOGRÁFICAS}

1. Cubilla, A. L. Fines de la Universidad: La investigación como función central. Universidad en el Paraguay. Desafíos y dilemas. 2000;71-81.

2. Taype-Rondán Á, Luque Bustamante L. Producción científica en Scopus de la Universidad de San Martín de Porres, Lima, Perú. Horiz Méd. 2014;14(4):37-42.

3. Mondragón-Cardona Á, Jiménez-Canizales CE, Alzate-Carvajal V. Oportunidades y desarrollo en las sociedades científicas estudiantiles. Cienc E Investig Medico Estud Latinoam [Internet]. 2013 [cited 2014 Aug 5];17(1) Available from: http://www.cimel.felsocem.net/index.php/CIMEL/article/ viewArticle/231
4. Alberto Perales. La investigación en la Formación Médica. Anales de la Facultad de Medicina, Universidad Nacional Mayor de San Marcos [Internet]. 1999 [cited 2016 Mar 27];55(3). Available from: http://sisbib.unmsm. edu.pe/bvrevistas/anales/v59_n3/form_medica.htm

5. Salas SP, Rigotti A. Physicians in biomedical research in Chile: A species under risk of extinction? Rev Médica Chile. 2005;133(1):121-8.

6. Rojas-Revoredo V. Las publicaciones en revistas indexadas, único indicador de la producción de las sociedades científicas estudiantiles. CIMEL. 2007;12(1):5-6. 